\title{
New Composites CP@AC Based on Lanthanide Succinates
}

\author{
Guilherme C. Santos, ${ }^{a}$ Carlos A. F. de Oliveira, ${ }^{b}$ Fausthon F. da Silva ${ }^{\circledR *, c}$ and \\ Severino Alves $\mathrm{Jr} . * a$ \\ ${ }^{a}$ Departamento de Química Fundamental, Universidade Federal de Pernambuco (UFPE), \\ 55067-901 Recife-PE, Brazil \\ ${ }^{b}$ Instituto Federal de Educação, Ciência e Tecnologia da Paraíba (IFPB), \\ 58015-020 João Pessoa-PB, Brazil \\ 'Departamento de Química, Universidade Federal da Paraíba (UFPB), \\ 58051-900 João Pessoa-PB, Brazil
}

\begin{abstract}
Composites derived from coordination polymers have been gathering great attention due to their singular properties and applications, especially catalysis and adsorption. In this work, seven new composites based on activated carbon (AC) and lanthanide-succinates ( $\mathrm{LnSuc}$; $\mathrm{Ln}=\mathrm{Pr}, \mathrm{Nd}, \mathrm{Sm}, \mathrm{Gd}$, Dy, Er and Tm, and Suc = succinate) 3D-coordination polymers were obtained using hydrothermal reactions. Coordination polymers in LnSuc@AC composites ( $\mathrm{Ln}=\mathrm{Pr}, \mathrm{Nd}, \mathrm{Sm}, \mathrm{Gd})$ crystallize in monoclinic system and $\mathrm{C} 2 / \mathrm{c}$ space group. However, the LnSuc in composites with Dy, Er and Tm have different crystal structures, due to the influence of the carbon matrix in the reaction. The systems were also characterized by Fourier transform infrared (FTIR) and thermogravimetric (TGA) analysis, and the results are in agreement with the X-ray powder diffraction (XRPD) data. The scanning electron microscopy (SEM) images confirm the crystallization of the compounds inside the pores of the carbon material. Photophysical properties of the NdSuc@AC and DySuc@AC were also investigated.
\end{abstract}

Keywords: coordination polymers, luminescence and lanthanide composites

\section{Introduction}

Coordination polymers (CPs) are crystalline solids obtained by the reaction between metal ions (and/or metal clusters) and multidentate organic ligands, with metal-ligand bonds extending in $1 \mathrm{D}, 2 \mathrm{D}$ and $3 \mathrm{D}^{1,2}$ These solids may present cavities, channels or interlamellar spaces, thus, hosting chemical species in those empty spaces, allowing applications in several fields such as luminescent ${ }^{3}$ and magnetic ${ }^{4}$ materials, electrical conductivity, ${ }^{5}$ adsorption, ${ }^{6}$ catalysis, ${ }^{7}$ and so on.

On the other hand, composites are strategically interesting due to the possibility of combining properties of different materials. In general, the poor chemical stability of the CPs limits their uses, nevertheless, CP-based composites show distinguished and different properties in comparison with the individual components. Many composite materials based on CPs have been prepared in the literature, ${ }^{8-10}$ such as

*e-mail: fausthon@quimica.ufpb.br; salvesjr@ufpe.br nanocomposites with metallic nanoparticles ${ }^{11}$ and systems based on carbonaceous materials, as carbon nanofibers. ${ }^{9,12}$

Our research group has explored with success the potential of lanthanide-based systems in many fields like luminescent devices, ${ }^{13}$ printable inks, ${ }^{14}$ matrices for solidstate extraction ${ }^{15,16}$ and so on. Recently, we have investigated the insertion of CPs crystals into activated carbon (AC) pores (metal-organic frameworks (MOFs)@ AC). ${ }^{17}$ Crystals of $\left[\mathrm{Ln}_{2}\left(\mathrm{Suc}_{3}\left(\mathrm{H}_{2} \mathrm{O}\right)_{2}\right] \cdot 0 \cdot 5 \mathrm{H}_{2} \mathrm{O}(\mathrm{Ln}=\mathrm{Eu}\right.$ and $\mathrm{Tb}$; $\mathrm{Suc}=$ succinate) were inserted in $\mathrm{AC}$ using hydrothermal reaction, in different mass ratios (1 to $50 \%) .{ }^{17}$ These new materials presented good performances for aldicarb detoxification in vivo via adsorption process. ${ }^{17} \mathrm{In}$ another work, we also demonstrated the synthesis and characterization of $\mathrm{Ln}_{2}(1 \text {,4-benzenedicarboxylate })_{3} \cdot\left(\mathrm{H}_{2} \mathrm{O}\right)_{4}$ ( LnBDC; $\mathrm{Ln}=\mathrm{Eu}$ and $\mathrm{Gd}) @ \mathrm{AC}$ composites and their selective dyes adsorption in different $\mathrm{pH}$ values. ${ }^{18}$ Thus, this type of CP-composite seems to improve the chemical and physical properties of $\mathrm{AC}$, showing great potential as adsorbent material. 
In this way, the main goal of this work is the synthesis of new lanthanide-succinates ( $\mathrm{LnSuc}$; $\mathrm{Ln}=\mathrm{Pr}, \mathrm{Nd}, \mathrm{Sm}$, Gd, Dy, Er and Tm)@AC composites. Composites were characterized by scanning electron microscopy (SEM) and energy-dispersive spectroscopy (EDS) and X-ray powder diffraction (XRPD). Results indicate the crystallization of the CPs within the AC pores, and the crystalline phases obtained in the formation of the composites were identified. Materials were also characterized by Fourier transform infrared spectroscopy (FTIR) and thermogravimetry (TGA), both in agreement with the crystalline structure inside the carbon matrix. The luminescent properties of composites with dysprosium and neodymium were also investigated.

\section{Experimental}

General

All reagents were obtained commercially and used without further purification. Succinic acid and the respective $\mathrm{Ln}_{2} \mathrm{O}_{3}(\mathrm{Ln}=\mathrm{Nd}, \mathrm{Sm}, \mathrm{Gd}, \mathrm{Dy}, \mathrm{Er}$ and Tm) and $\mathrm{Pr}_{3} \mathrm{O}_{5}$ were obtained from Sigma-Aldrich (99\% purity). The AC was obtained from the Dinâmica Química. Lanthanide chlorides $\mathrm{LnCl}_{3} \cdot 6 \mathrm{H}_{2} \mathrm{O}(\mathrm{Ln}=\mathrm{Pr}, \mathrm{Nd}, \mathrm{Sm}$, $\mathrm{Gd}$, Dy, Er and Tm) were synthesized by reaction of hydrochloric acid with the corresponding lanthanide oxide. ${ }^{13}$

Synthesis of LnSuc ( $L n=N d, S m, G d$, Dy, Er and Tm)

An amount of $0.059 \mathrm{~g}(0.5 \mathrm{mmol})$ of succinic acid was placed in $10 \mathrm{~mL}$ of deionized water in a Teflon vessel $(23 \mathrm{~mL})$. The $\mathrm{pH}$ was adjusted to $5-6$ by the addition of sodium hydroxide $\left(2.5 \mathrm{mmol} \mathrm{L}^{-1}\right)$. After that, $0.5 \mathrm{mmol}$ of the respective lanthanide chloride hydrate was added to the reaction mixture. The system was sealed and heated to $120^{\circ} \mathrm{C}$ for $96 \mathrm{~h}$. Then, the system was cooled to room temperature. Crystals were collected by filtration, washed with distilled water and acetone, and air-dried. The reaction yields ranged from $39 \%$ for the Dy compound to $68 \%$ for the $\mathrm{Nd}$ compound.

\section{Synthesis of the LnSuc@AC composites}

The synthesis procedures of the composites were identical to the synthesis of the respective CPs, except for the addition of $\mathrm{AC}$ (equivalent to $50 \%$ in the sum of the starting reagents masses, succinic acid and lanthanide salt), for each reaction. The solids obtained were washed with distilled water and air-dried.

\section{Physical measurements}

The FTIR spectroscopy was carried out with a Bruker FTIR IFS66 spectrometer, in range of 4000-400 $\mathrm{cm}^{-1}$, using $\mathrm{KBr}$ pellets. The TGA curves were obtained in a Shimadzu 60-H TGA analyzer under $\mathrm{N}_{2}$ atmosphere, from room temperature to $1000{ }^{\circ} \mathrm{C}$, at $10{ }^{\circ} \mathrm{C} \mathrm{min}{ }^{-1}$. The XRPD analyses were performed at room temperature in a Shimadzu XRD-700 diffractometer with $\mathrm{CuK}_{\alpha}(1.57 \AA)$ source, scanning $0.01^{\circ} \mathrm{s}^{-1}$, between $5-50^{\circ}$. The SEM-EDS images were obtained in a Shimadzu SS550 microscope with tungsten filament, working at $20 \mathrm{kV}$ and a $9.8-10 \mathrm{~mm}$ working distance (WD). The photoluminescence spectra were obtained in a Horiba Jobin-Yvon Fluorolog-3 modular spectrofluorometer with double monochromator, using a $450 \mathrm{~W}$ xenon lamp.

\section{Results and Discussion}

\section{Characterization of the LnSuc systems}

The LnSuc system has been extensively studied in the literature, and compounds with structural and stoichiometric diversity can be found. ${ }^{19-25}$ For the synthesis of the LnSuc ( $\mathrm{Ln}=\mathrm{Pr}, \mathrm{Nd}, \mathrm{Sm}, \mathrm{Gd}, \mathrm{Dy}, \mathrm{Er}$ and Tm) systems, the methodology of hydrothermal synthesis was used resulting in single crystals with yield ranging from 68 (for PrSuc) to 39\% (for DySuc). Figure 1 shows the optical microscopy images of some crystals obtained in the synthesis.

Compounds obtained are isostructural (as observed in the X-ray diffraction patterns described below) and their structures are well described in the literature..$^{20} \mathrm{All}$ products crystallize in the monoclinic system with $\mathrm{C} 2 / \mathrm{c}$ space group and chemical composition $\left[\mathrm{Ln}_{2}(\mathrm{Suc})_{3}\left(\mathrm{H}_{2} \mathrm{O}\right)_{2}\right] \cdot 0 \cdot 5 \mathrm{H}_{2} \mathrm{O}$ $(\mathrm{Ln}=\mathrm{Pr}, \mathrm{Nd}, \mathrm{Sm}, \mathrm{Gd}, \mathrm{Dy}, \mathrm{Er}$ and Tm). Lanthanide trivalent ions are nine-coordinated by eight oxygen atoms from succinate anions and one from a coordinated water molecule, with tricapped trigonal prism geometry. ${ }^{20}$

FTIR spectra of the LnSuc systems (Figure S1, Supplementary Information (SI) section) show bands related to the ligand and the water molecules present in the structure. The similarity also indicates that all compounds have the same structure. The broad band centered at $3350 \mathrm{~cm}^{-1}$ is regarding to the asymmetric stretching of the $\mathrm{O}-\mathrm{H}$ groups and confirms the presence of water molecules.

Signals located between 2981 and $2921 \mathrm{~cm}^{-1}$ are related to the methylene $\left(\mathrm{CH}_{2}\right)$ groups of the ligands. The intense peaks at 1620 and $1460 \mathrm{~cm}^{-1}$ are related to the asymmetric and symmetric of the carboxyl groups, shifted in comparison to the free succinic acid due to coordination 

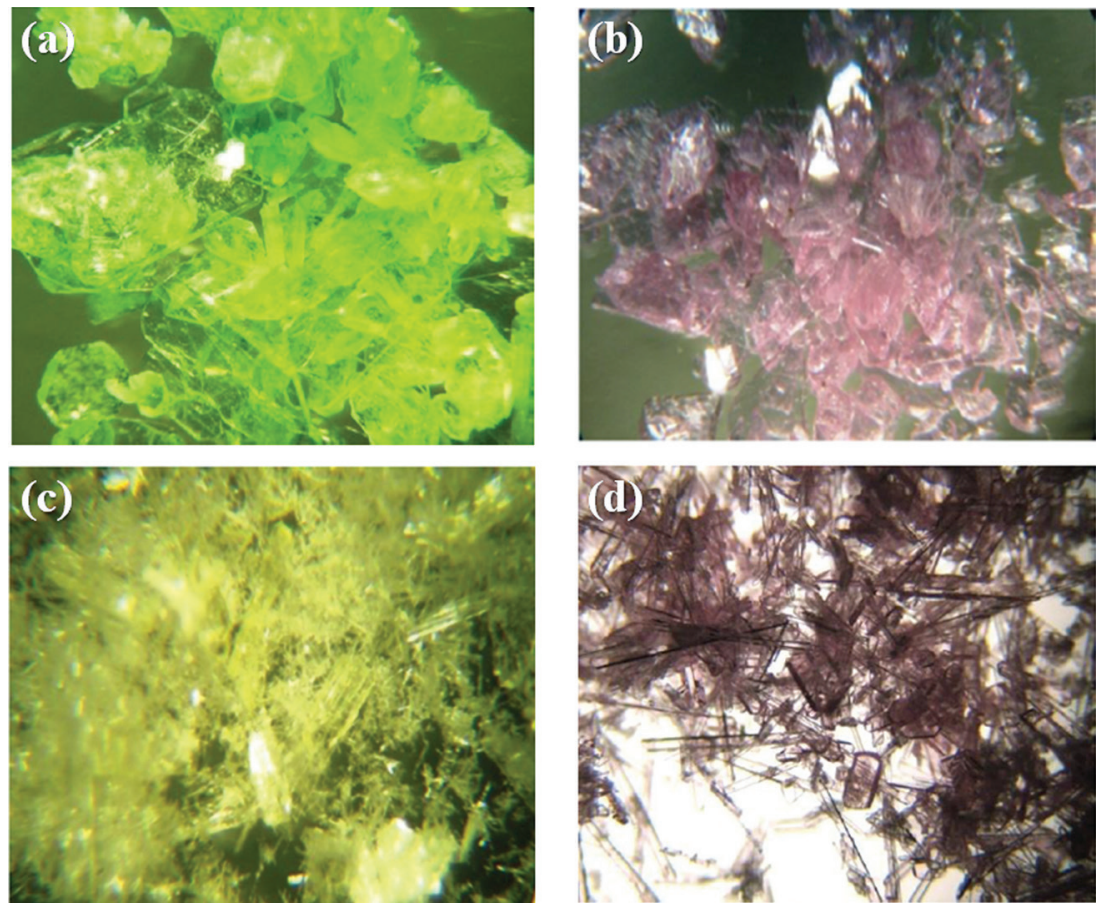

Figure 1. Optical microscopy images of (a) PrSuc, (b) NdSuc, (c) SmSuc and (d) ErSuc.

with the metal cations. All the signals observed are in agreement with the literature. ${ }^{21,26}$

Powder patterns of the LnSuc systems are shown in Figure 2. A good correlation in comparison with the diffraction pattern of the monoclinic phase already reported in the literature ${ }^{20}$ was observed. The absence of additional peaks indicates high purity and no formation of secondary crystalline phases. In all cases, the most intense signal was observed near $10^{\circ}$ related to the (002) diffraction plane.

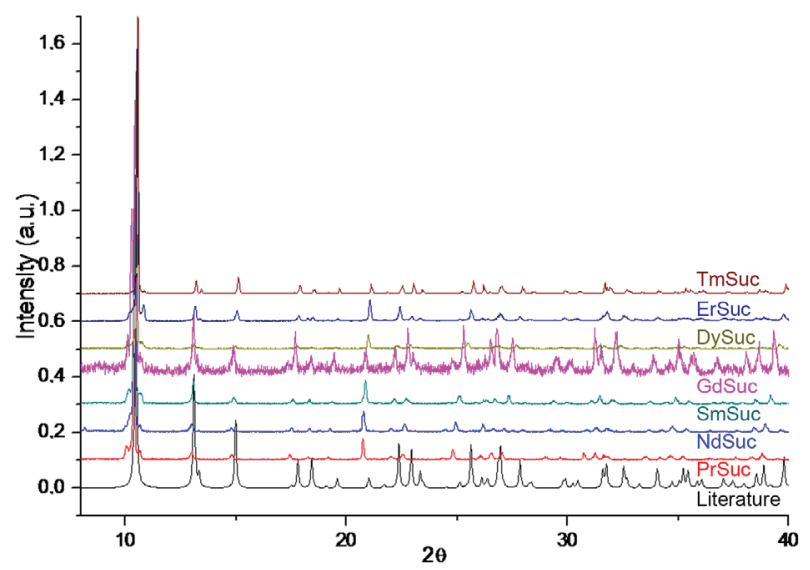

Figure 2. Powder patterns of the LnSuc systems in comparison with the already reported structure..$^{20}$

\section{Characterization of LnSuc@AC composites}

LnSuc@AC composites were obtained under the same synthetic conditions of the respective LnSuc CP, except by the insertion of $50 \%(\mathrm{~m} / \mathrm{m})$ of AC in the reaction system. The SEM images (Figure 3) of composites compared to the free AC indicate the in situ crystallization of LnSuc inside the pores of the carbonaceous material. EDS analyses inside and outside the composite pores confirm the chemical composition of the materials (Figures S2-S15, SI section). The results are similar to the composites containing europium and terbium succinates, recently reported by our research group..$^{17}$

XRPD patterns of the composites (Figure 4) were used to identify the crystalline phases inserted into the AC pores. For the composites containing $\mathrm{Pr}, \mathrm{Nd}, \mathrm{Sm}$ and $\mathrm{Gd}$, the same signals compared with the respective LnSuc CP were observed, indicating the same crystalline structures were obtained in the reaction with the presence of AC. Regarding these composites, the SmSuc@AC presents higher crystallinity in comparison with LnSuc@ $@ \mathrm{AC}(\mathrm{Ln}=\mathrm{Pr}$, Nd and $\mathrm{Gd}$ ) and higher preferential orientation in the (002) plane. The amorphous band observed in LnSuc@AC $(\mathrm{Ln}=\mathrm{Pr}, \mathrm{Nd}$ and $\mathrm{Gd})$ is related to the carbon matrix.

In the diffractogram of ErSuc@AC and TmSuc@AC additional signals are observed, indicating secondary phases. In the case of DySuc@ AC composite, a different diffraction pattern was observed, in comparison to the crystalline phase DySuc obtained without the presence of AC under the same conditions, and a new crystalline structure was inserted into the pores of AC. The new crystalline phases found in these composites, to the best of our knowledge, were not reported in the literature up to the 

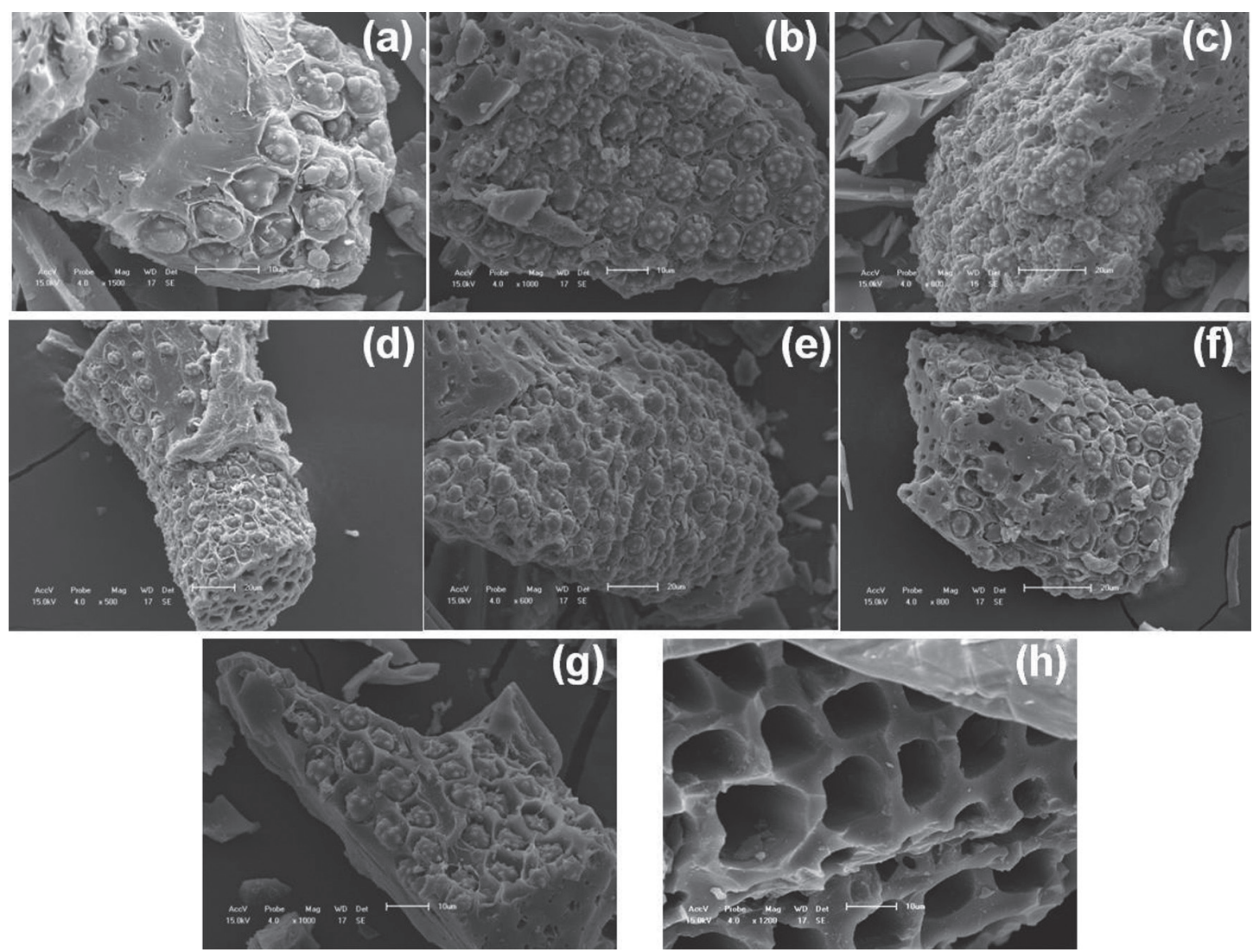

Figure 3. SEM images of LnSuc@ AC composites (Ln = (a) Pr; (b) Nd; (c) Sm; (d) Gd; (e) Dy; (f) Er and (g) Tm) and (h) AC.

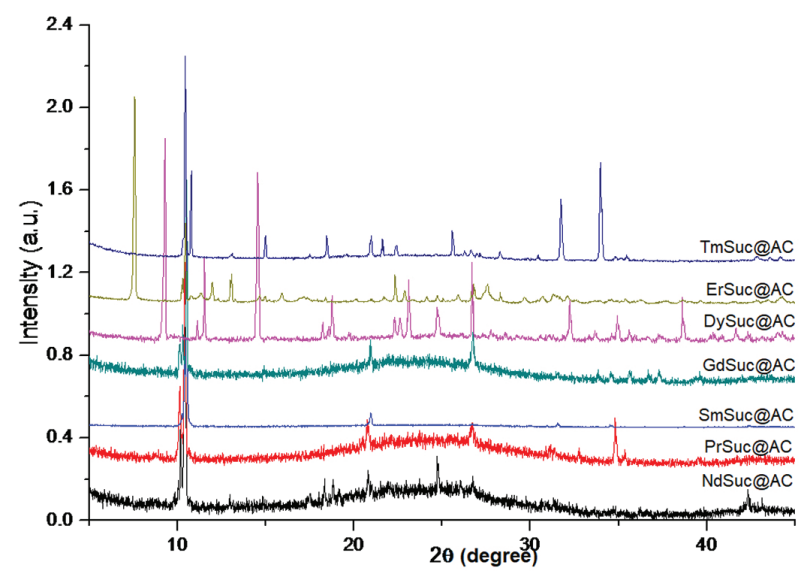

Figure 4. XRPD patterns of the LnSuc@AC composites.

present moment. In this way, we observe the influence of the coal in the crystallization of the Ln/Suc system with the increase of the lanthanide contraction. Recent exploratory studies have demonstrated the role of organic molecules in the formation of different $\mathrm{Ln} / \mathrm{Suc}$ structures. ${ }^{19}$ Thus, in our case, the coal must be acting in a similar way, as modulator and/or inducer for the formation of new crystalline structures. However, more detailed studies should be conducted to investigate this experimental observation, being far from the scope of this work.
ForDySuc@AC,ErSuc@AC and TmSuc@AC composites, the infrared spectra are shown in Figure 5. All signals are related to succinate anions, indicating that only this ligand is inserted into the structure of material. The peaks near $3600 \mathrm{~cm}^{-1}$ are related to the presence of free $\mathrm{O}-\mathrm{H}$ groups. Bands between 1650 and $1300 \mathrm{~cm}^{-1}$ correspond to the symmetrical and asymmetrical stretching signals of the carboxyl group $(\mathrm{C}=\mathrm{O}$ and $\mathrm{C}-\mathrm{O}$, respectively). Changes in the number and position of the carboxyl group bands in comparison with the respective free lanthanide succinate were also observed. This could be related to a change in the coordination mode between succinate anions and lanthanide ions in these compounds, which is in agreement with the results of X-ray diffraction. Infrared spectra of the other composites are similar to the respective LnSuc CP and are shown in Figure S16 (SI section).

TGA curves for LnSuc@AC composites $(\mathrm{Ln}=\mathrm{Pr}, \mathrm{Nd}$, $\mathrm{Sm}$ and $\mathrm{Gd}$ ) are shown in Figure 6. All materials show an initial mass loss between room temperature and $70{ }^{\circ} \mathrm{C}$, related to adsorbed species. After 100 until $210{ }^{\circ} \mathrm{C}$, the loss of hydrated and coordinated water molecules took place. All results are similar to the compounds without the presence of AC. ${ }^{19-26}$

For the composite containing Dy, Er and Tm, TGA are shown in Figure 7. Again, the initial loss mass until $70{ }^{\circ} \mathrm{C}$ 


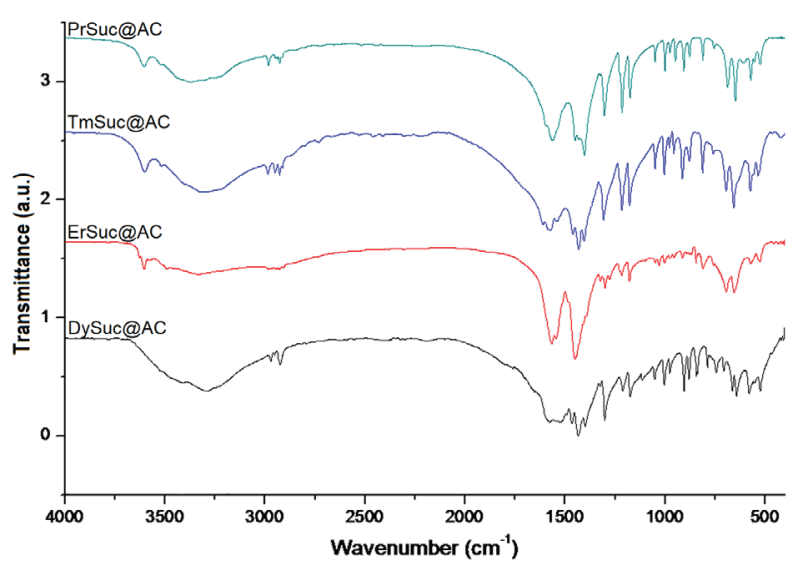

Figure 5. FTIR spectra of the DySuc@AC,ErSuc@AC, TmSuc@AC and PrSuc@AC composites.

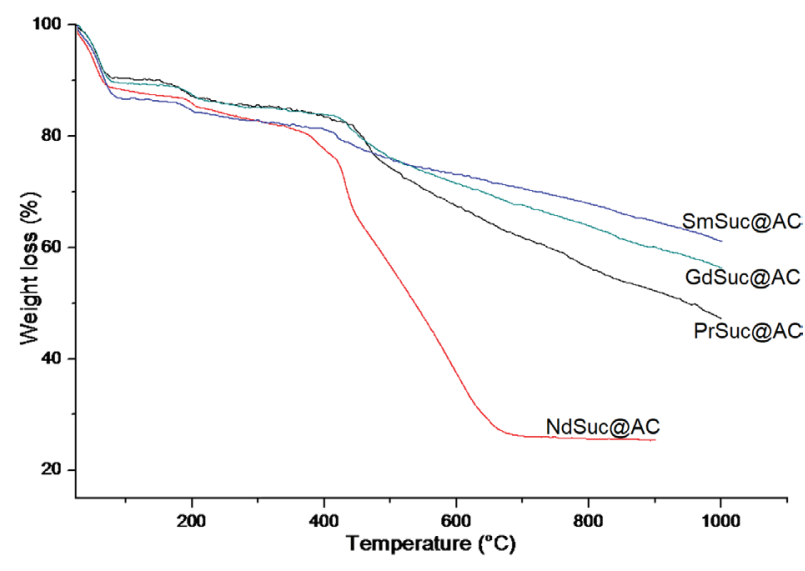

Figure 6. Thermogravimetric curves of LnSuc@ AC composites ( $\mathrm{Ln}=\mathrm{Pr}$, $\mathrm{Nd}, \mathrm{Sm}$ and $\mathrm{Gd}$ ).

was observed, due to the adsorbed species. The events related with water molecules (coordinated and hydrated) were also noticed, but at lower temperatures, indicating lower thermal stability for these materials.

The photoluminescence spectra of NdSuc (Figure S17, SI section) show $4 \mathrm{f}$-transitions characteristic of the

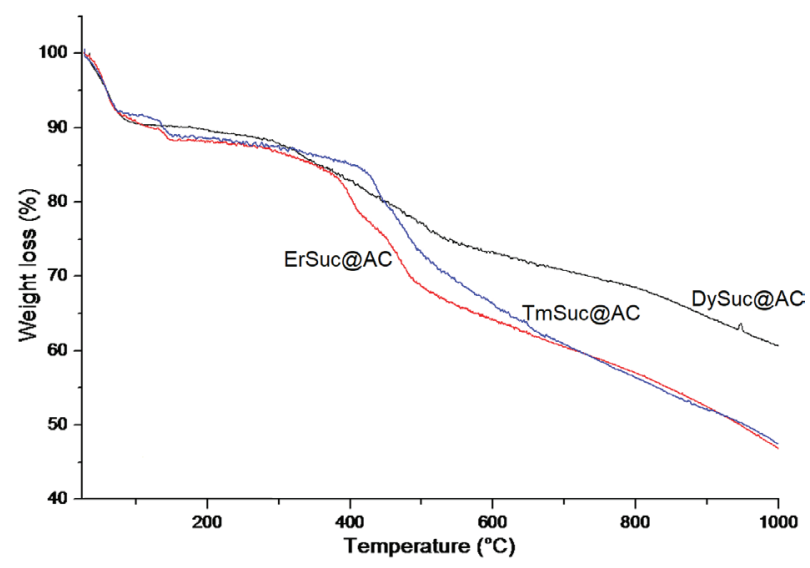

Figure 7. Thermogravimetric curves of LnSuc@ AC composites (Ln=Dy, Er and $\mathrm{Tm})$. excitation and emission centred in the $\mathrm{Nd}^{3+}$ ions..$^{27,28}$ In the excitation spectrum, the most intense peak located at $353 \mathrm{~nm}$ is related to the ${ }^{4} \mathrm{I}_{11 / 2} \rightarrow\left({ }^{4} \mathrm{D}_{5 / 2} ;{ }^{4} \mathrm{D}_{3 / 2} ;{ }^{4} \mathrm{D}_{1 / 2}\right)$ electronic transition. The sample was irradiated at $353 \mathrm{~nm}$ and the emission spectrum was collected (Figure S17, SI section). Two typical transitions of the $\mathrm{Nd}^{3+}$ ions in the infrared region were observed at 1055 and $1327 \mathrm{~nm}$, regarding the ${ }^{4} \mathrm{~F}_{3 / 2} \rightarrow{ }^{4} \mathrm{I}_{11 / 2}$ and ${ }^{4} \mathrm{~F}_{3 / 2} \rightarrow{ }^{4} \mathrm{I}_{13 / 2}$ transitions, respectively. ${ }^{27,28}$

The excitation spectrum of the NdSuc@AC, monitoring the emission at $1055 \mathrm{~nm}$, is shown in Figure 8. Fewer transitions were observed, due to the loss of energy related to the vibrational modes of the carbon matrix. Again, the most intense signal is related to the transition ${ }^{4} \mathrm{I}_{1 / 2} \rightarrow\left({ }^{4} \mathrm{D}_{5 / 2} ; \mathrm{D}_{3 / 2} ; \mathrm{D}_{1 / 2}\right)$. The sample was excited at $350 \mathrm{~nm}$, and a similar profile in the emission spectrum was observed (Figure 8), in comparison to the free CP.

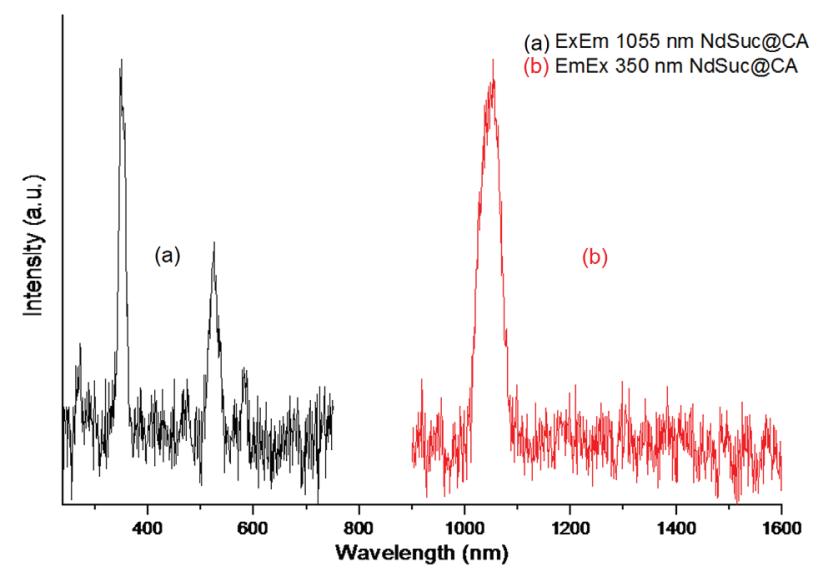

Figure 8. (a) Excitation spectrum and (b) emission spectrum of NdSuc@AC.

In the photoluminescence spectra of DySuc (Figure S18, SI section), all typical signals related to the $\mathrm{Dy}^{3+}$ ions were observed. ${ }^{29,30}$ The highest peak at $365 \mathrm{~nm}$ is regarding the transition from the ground state ${ }^{6} \mathrm{H}_{15 / 2}$ to the excited state ${ }^{4} \mathrm{I}_{13 / 2}$. The emission spectrum $\left(\lambda_{\text {exc }}=350 \mathrm{~nm}\right)$ shows an intense signal at $574 \mathrm{~nm}$, due to the transition ${ }^{4} \mathrm{~F}_{9 / 2} \rightarrow{ }^{6} \mathrm{H}_{13 / 2}$. In the case of DySuc@AC (Figure 9), a very similar photoluminescence profile was observed. The signals of the $\mathrm{Dy}^{3+}$ ions are overlapped by an emission in the blue region, probably due to the carbon matrix.

\section{Conclusions}

In this work, seven new LnSuc@AC composites were obtained. The XRPD and infrared spectroscopy indicate the same crystalline phase for composites with $\mathrm{Pr}, \mathrm{Nd}$, $\mathrm{Sm}$ and Gd. The formation of new structure for systems with Dy, Er and Tm could be related with the influence of $\mathrm{AC}$ during the reaction. The photoluminescence properties 


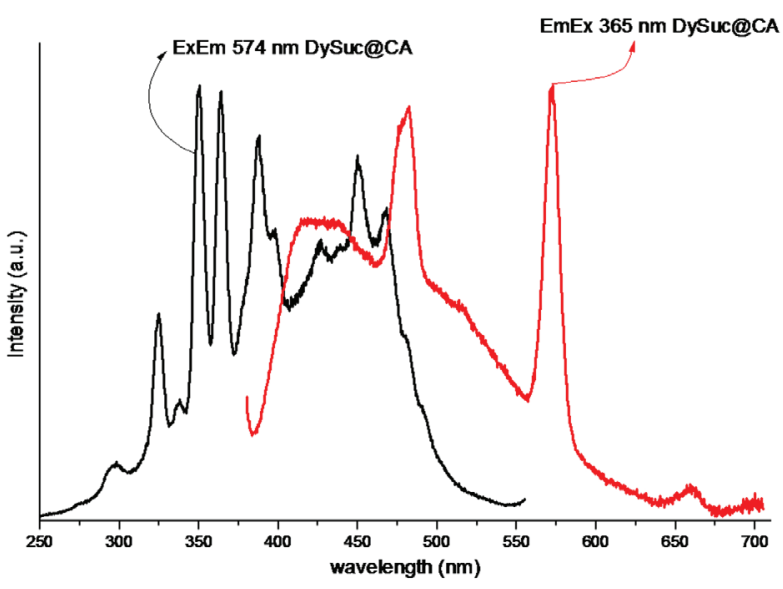

Figure 9. Excitation spectrum (black line) and emission spectrum (red line) of DySuc@AC.

of the composites indicate the main signals regarding the 4f-transition of the lanthanide trivalent ions.

\section{Supplementary Information}

Supplementary data (FTIR and SEM-EDS) are available free of charge at http://jbcs.sbq.org.br as PDF file.

\section{Acknowledgments}

The authors would like to thank CAPES, FACEPE and CNPq for the financial support, and Mrs Leonis Luz and Mrs Yago Rodrigues for their scientific collaboration.

\section{References}

1. Férey, G.; Chem. Soc. Rev. 2008, 37, 191.

2. Rowsell, J. L. C.; Yaghi, O. M.; Microporous Mesoporous Mater. 2004, 73, 3 .

3. Kurmoo, M.; Chem. Soc. Rev. 2009, 38, 1353.

4. Rocha, J.; Carlos, L. D.; Almeida Paz, F. A.; Ananias, D.; Chem. Soc. Rev. 2011, 40, 926.

5. Givaja, G.; Amo-Ochoa, P.; Gómez-García, C. J.; Zamora, F.; Chem. Soc. Rev. 2012, 41, 115.

6. Li, J.-R.; Kuppler, R. J.; Zhou, H.-C.; Chem. Soc. Rev. 2009 , $38,1477$.

7. Zhu, L.; Liu, X.-Q.; Jiang, H.-L.; Sun, L.-B.; Chem. Rev. 2017, 117,8129

8. Zhang, Y.; Feng, X.; Yuan, S.; Zhou, J.; Wang, B.; Inorg. Chem. Front. 2016, 3, 896

9. Liu, X.-W.; Sun, T.-J.; Hu, J.-L.; Wang, S.-D.; J. Mater. Chem. A 2016, 4, 3584.

10. Zhu, Q.-L.; Xu, Q.; Chem. Soc. Rev. 2014, 43, 5468.

11. Meilikhov, M.; Yusenko, K.; Esken, D.; Turner, S.; van Tendeloo, G.; Fischer, R. A.; Eur. J. Inorg. Chem. 2010, 24, 3701.
12. Pachfule, P.; Balan, B. K.; Kurungot, S.; Banerjee, R.; Chem. Commun. 2012, 48, 2009.

13. Lima, P. P.; Malta, O. L.; Alves Jr., S.; Quim. Nova 2005, 28, 805.

14. da Luz, L. L.; Milani, R.; Felix, J. F.; Ribeiro, I. R. B.; Talhavini, M.; Neto, B. A. D.; Chojnacki, J.; Rodrigues, M. O.; Júnior, S. A.; ACS Appl. Mater. Interfaces 2015, 7, 27115.

15. Carvalho, P. H. V.; Barreto, A. S.; Rodrigues, M. O.; Prata, V. M.; Alves, P. B.; Mesquita, M. E.; Alves Jr., S.; Navickiene, S.; J. Sep. Sci. 2009, 32, 2132.

16. Aquino, A.; Wanderley, K. A.; Paiva-Santos, C. O.; de Sá, G. F.; Alexandre, M. R.; Alves Jr., S.; Navickiene, S.; Talanta 2010, 83,631 .

17. Oliveira, C. A. F.; da Silva, F. F.; Jimenez, G. C.; Silva Neto, J. F.; Souza, D. M. B.; Souza, I. A.; Alves Jr., S.; Chem. Commun. 2013, 49, 6486.

18. Santos, G. C.; Barros, A. L.; Oliveira, C. A. F.; da Luz, L. L.; da Silva, F. F.; Demets, G. J.-F.; Alves Jr., S.; PLoS One 2017, 12, e0170026.

19. D’Vries, R. F.; Camps, I.; Ellena, J.; Cryst. Growth Des. 2015 , 15,3015 .

20. Manna, S. C.; Zangrando, E.; Bencini, A.; Benelli, C.; Chaudhuri, N. R.; Inorg. Chem. 2006, 45, 9114.

21. Oliveira, C. A. F.; da Silva, F. F.; Malvestiti, I.; Malta, V. R. S.; Dutra, J. D. L.; da Costa Jr., N. B.; Freire, R. O.; Alves Jr., S.; J. Solid State Chem. 2013, 197, 7.

22. Bernini, M. C.; Gándara, F.; Iglesias, M.; Snejko, N.; GutiérrezPuebla, E.; Brusau, E. V.; Narda, G. E.; Monge, M. A.; Chem. - Eur. J. 2009, 15, 4896.

23. Zhang, H.-T.; Song, Y.; Li, Y.-X.; Zuo, J.-L.; Gao, S.; You, X.-Z.; Eur. J. Inorg. Chem. 2005, 4, 766.

24. Rahahlia, N.; Benmerad, B.; Guehria-Laidoudi, A.; Dahaoui, S.; Lecomte, C.; J. Mol. Struct. 2007, 833, 42.

25. Serpaggi, F.; Férey, G.; Microporous Mesoporous Mater. 1999, $32,311$.

26. Bernini, M. C.; Brusau, E. V.; Narda, G. E.; Pozzi, C. G.; Punte, G.; Lehmann, C. W.; Eur. J. Inorg. Chem. 2007, 5, 684.

27. Gorni, G.; Valazquez, J. J.; Mather, G. C.; Duran, A.; Chen, G.; Sundararajan, M.; Balda, R.; Fernandez, J.; Pascual, M. J.; J. Eur. Ceram. Soc. 2017, 37, 1695.

28. Kalinovskaya, I. V.; Mamaev, A. Y.; Karasev, V. E.; Russ. J. Gen. Chem. 2011, 81, 1407.

29. Chepyga, L. M.; Hertle, E.; Ali, A.; Zigan, L.; Osvet, A.; Brabec, C. J.; Batentschuk, M.; J. Lumin. 2018, 197, 23.

30. Bui, A. T.; Roux, A.; Grichine, A.; Duperray, A.; Andraud, C.; Maury, O.; Chem. - Eur. J. 2018, 24, 3408.

Submitted: July 23, 2018 Published online: November 6, 2018 